OPEN ACCESS

Edited by:

Philippe De Deurwaerdere,

Université de Bordeaux, France

Reviewed by:

Laurent Juvin,

Université de Bordeaux, France

Farinaz Nasirinezhad,

Iran University of Medical

Sciences, Iran

*Correspondence:

Florence E. Perrin

florence.perrin@inserm.fr

${ }^{+}$These authors have contributed equally to this work

Specialty section: This article was submitted to

Neuropharmacology,

a section of the journal

Frontiers in Pharmacology

Received: 07 October 2020 Accepted: 14 January 2021

Published: 10 February 2021

Citation:

Lattard A, Poulen G, Bartolami S,

Gerber YN and Perrin FE (2021)

Negative Impact of Sigma-1 Receptor Agonist Treatment on Tissue Integrity and Motor Function Following Spinal

Cord Injury.

Front. Pharmacol. 12:614949.

doi: 10.3389/fphar.2021.614949

\section{Negative Impact of Sigma-1 Receptor Agonist Treatment on Tissue Integrity and Motor Function Following Spinal Cord Injury}

\author{
Alise Lattard ${ }^{1 \dagger}$, Gaëtan Poulen ${ }^{1,2 t}$, Sylvain Bartolami ${ }^{1}$, Yannick N. Gerber ${ }^{1 \dagger}$ and \\ Florence E. Perrin ${ }^{1,3 * t}$ \\ ${ }^{1} \mathrm{MMDN}$, University of Montpellier, EPHE, INSERM, Montpellier, France, ${ }^{2}$ Department of Neurosurgery, CHU, Montpellier, France, \\ ${ }^{3}$ Institut Universitaire de France (IUF), University of Montpellier, INSERM U1198, Montpellier, France
}

In traumatic spinal cord injury, the initial trauma is followed by a cascade of impairments, including excitotoxicity and calcium overload, which ultimately induces secondary damages. The sigma-1 receptor is widely expressed in the central nervous system and is acknowledged to play a key role in calcium homeostasis. Treatments with agonists of the sigma-1 receptor induce beneficial effects in several animal models of neurological diseases. In traumatic injury the use of an antagonist of the sigma-1 receptor reversed several symptoms of central neuropathic pain. Here, we investigated whether sigma-1 receptor activation with $\mathrm{PRE}-084$ is beneficial or detrimental following $\mathrm{SCl}$ in mice. First, we report that PRE-084 treatment after injury does not improve motor function recovery. Second, using ex vivo diffusion weighted magnetic resonance imaging completed by histological analysis, we highlight that $\sigma 1 \mathrm{R}$ agonist treatment after $\mathrm{SCl}$ does not limit lesion size. Finally, PRE-084 treatment following SCl decreases NeuN expression and increases astrocytic reactivity. Our findings suggest that activation of sigma-1 receptor after traumatic spinal cord injury is detrimental on tissue preservation and motor function recovery in mice.

Keywords: spinal cord injury, sigma 1 receptor, PRE-084, motor function, neuromuscular junction, gliosis

\section{INTRODUCTION}

Traumatic spinal cord injury (SCI) results in 0.6-0.9 million annual new cases worldwide (Kumar et al., 2018). SCI symptoms include sensory, motor and autonomic deficits ranging from minimal dysfunctions to complete tetraplegia. Following the primary traumatic mechanical disruption of spinal cord tissues, a cascade of events including vascular impairment, mitochondrial dysfunction, excitotoxicity and calcium overload characterizes the secondary phase of injury and further exacerbates the lesion. There is currently no curative treatment to improve neurological recovery after SCI.

The sigma-1 $(\sigma 1)$ receptor is a unique non-G-protein-coupled membrane-associated protein ubiquitously expressed with chaperone activity (Hayashi and Su, 2007). The central nervous system (CNS) is one of the major sites of $\sigma 1 \mathrm{R}$ activity. Indeed, $\sigma 1 \mathrm{R}$ is expressed in neurons, astrocytes, oligodendrocytes and microglia and is found at the endoplasmic reticulum (ER) membrane that is associated with the mitochondria (Mitochondria-Associated ER Membranes [MAMs]) [for review see (Penke et al., 2018)]. $\sigma 1 \mathrm{R}$, that forms a complex at MAMs with BiP 
(binding immunoglobulin protein), another chaperone protein, is a $\mathrm{Ca}^{2+}$ sensitive and ligand-operated receptor chaperone at MAMs (Hayashi and Su, 2007). In fact, $\sigma 1 \mathrm{R}$ modulates $\mathrm{Ca}^{2+}$ exchange between ER and mitochondria by interacting with inositol-1,4,5 triphosphate receptors (IP3Rs) (Hayashi and Su, 2007). Following ligand stimulation, $\sigma 1 \mathrm{R}$ dissociates from $\mathrm{BiP}$, conducting to a lengthened $\mathrm{Ca}^{2+}$ signaling into mitochondria. Decreasing $\sigma 1 \mathrm{R}$ activity in cell enhances apoptosis, whereas increasing it neutralizes ER stress response (Hayashi and Su, 2007). o1R is amongst others implicated in neuroplasticity, neuroprotection and carcinogenesis (Hayashi and Su, 2007). Interestingly, newborn $\sigma 1 \mathrm{R}$ knockout mice, display a transient enhanced proliferation of progenitor cells in the hippocampal dentate gyrus followed by a decrease in survival and neurite outgrowth of newly generated neurons associated with a reduced function of $\mathrm{N}$-methyl-D-aspartate receptor (NMDAr) (Sha et al., 2013).

PRE-084 (2-(4-morpholinoethyl)-1-phenylcyclohexane-1carboxylate hydrochloride) is a highly selective $\sigma 1 \mathrm{R}$ agonist displaying minimal cross reactivity with other receptors [for extensive review see (Motawe et al., 2020)]. It had been shown in vivo that PRE-084 binds with $\sigma 1 \mathrm{R}$ and is rapidly distributed in the CNS (Motawe et al., 2020). Using excitotoxic injury in organotypic spinal cord slices, Guzmán-Lenis et al. demonstrated that PRE-084 not only induces a neuroprotective effect and decreases neuronal damage but also enhances axonal re-growth (Guzman-Lenis et al., 2009). Furthermore, $\sigma 1 \mathrm{R}$ activation attenuates several aspects of microglial activation in primary culture of rat microglia (Hall et al., 2009).

In vivo, the beneficial effects of PRE-084 has been reported in animal models of neurological diseases such as Alzheimer disease, Parkinson disease, amyotrophic lateral sclerosis, spinal muscular atrophy, Huntington disease, vascular dementia, amnesia, embolic stroke and multiple sclerosis. Though, PRE-084 did not improve outcomes following seizures [for review see (Motawe et al., 2020)].

To our knowledge, PRE-084 impact has not been investigate following acute traumatic injury in the CNS. However, it had been recently shown that after spinal cord contusion in mice, treatment with a $\sigma 1 \mathrm{R}$ antagonist reduced mechanical allodynia and thermal hyperalgesia that are characteristics of central neuropathic pain (Castany et al., 2018; Castany et al., 2019). On the one hand, treatment with $\sigma 1 \mathrm{R}$ agonist plays beneficial roles in chronic neurological diseases, including amelioration of motor-induced symptoms. On the other hand, treatment with $\sigma 1 \mathrm{R}$ antagonist induce positive outcomes on injury-induced pain following acute CNS injury. It is therefore of interest to investigate the role of $\sigma 1 \mathrm{R}$ activation on motor function recovery following SCI.

In this study, we assessed the effect of PRE-084 treatment following lateral hemisection of the mouse spinal cord on motor recovery and spinal cord structure at tissue and cellular levels.

\section{MATERIAL AND METHODS}

\section{Study Approval}

Experiments were approved by the French Ministry of National Education, Higher Education and Research, the regional ethic committee $n^{\circ} 36$ and by the Veterinary Services Department of Hérault (authorization $\mathrm{n}^{\circ} 34118$ ) and followed the European legislative, administrative and statutory measures (EU/ Directive/2010/63) and the ARRIVE guidelines.

\section{Spinal Cord Injury and Treatment}

We used female mice of 5 months of age on a C57BL6/6 background (Charles River, Wilmington US) housed in controlled environment (hygrometry 60\%, temperature $26-28^{\circ} \mathrm{C}$, 12:12 light/dark cycle, food and water ad libitum). Following weaning females were housed in groups of 6 . Three weeks prior surgery they were randomly affected to the untreated or treated groups. We used 3-4\% isoflurane (Vetflurane ${ }^{\circledR}$, Virbac, France) and $1 \mathrm{~L} / \mathrm{min}$ oxygen flow rate to induce the anesthesia and then $1-2 \%$ isoflurane to maintain it throughout the surgery. We applied eye gel to the cornea at the beginning of the surgery. After shaving, the skin was cleaned (Vetadine ${ }^{\circledR}$, Bayer, Australia). We performed a lateral spinal cord hemisection at thoracic 9 (T9) level to preserve complete respiratory function while obtaining a monoplegia. Hemisection was done using a micro knife (1031512, Fine Science Tools) after vertebral laminectomy. Muscles and skin were sutured and mice were left to recover on a temperaturecontrolled pad. Post-operative cares: Animals were monitored over 1-h following the surgery before returning to their cages. Bladders were emptied manually twice daily until recovery of full sphincter control and observation of potential signs of pain or distress was carefully done. None of the animal developed selfbiting, cutaneous infection or inflammation. We measured bodyweights prior to surgery and then daily until the study ends (Figure 1A). Treatment consisting in (2-(4morpholinoethyl)-1-phenylcyclohexane-1-carboxylate

hydrochloride) (PRE-084, Sigma-Aldrich, Saint-QuentinFallavier, France) diluted in the drinking water at a dose of $1 \mathrm{mg} / \mathrm{kg} /$ day, and given throughout 6 weeks after injury. To adjust and control the dose, we weighted the mice, we measured water consumption and we gave fresh PRE-084 solution twice a week.

Number of mice: six uninjured mice (three treated and three untreated) and 24 hemisected mice (12 treated and 12 untreated).

\section{Behavioral Analysis}

All tests were done during the light cycle (from 1 pm to $4 \mathrm{pm}$ ). Open field test was always done first and followed by CatWalk test with a time interval of $1 \mathrm{~h}$ max. Habituation to tests was done to avoid stress-induced bias; mice were placed on the CatWalk XT ${ }^{\circledR}$ glass plate as well as in the open field area for 20 min 7 days prior to the first recordings. Mice were then tested at seven- and 1-day prior injury followed by acquisitions at $72 \mathrm{~h}, 5$ days and then once a week up to 6 weeks after lesion. Open field (EthoTrack, InnovationNet, Tiranges, France) and CatWalk XT ${ }^{\circledast}$ (Noldus, Wageningen, 

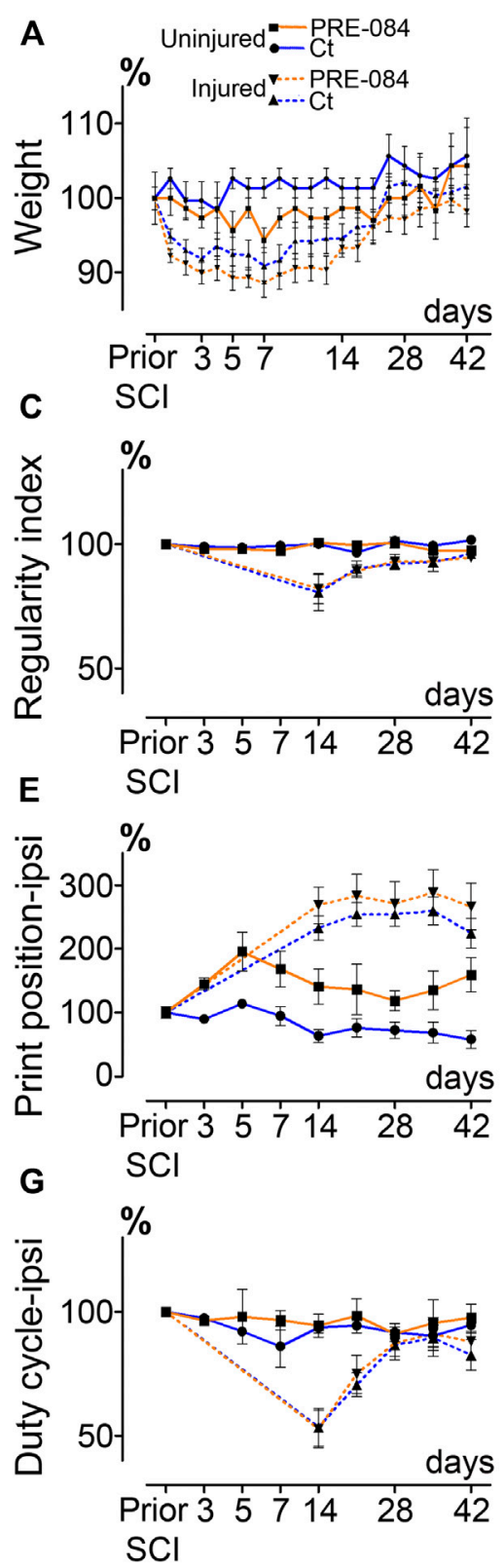

B
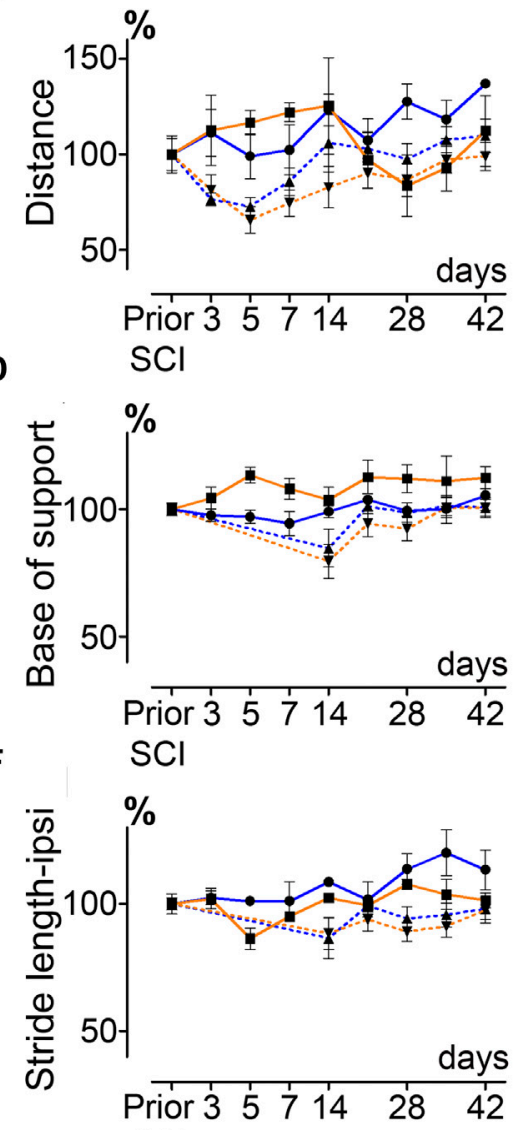

H

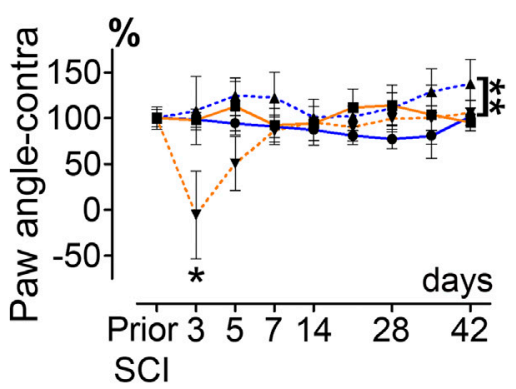

FIGURE 1 | Effects of PRE-084 treatment on spontaneous motor activity and gait patterns after SCl. Weight curves (A). Open field analysis of the distance covered in 8 min by mice in all groups (B). Walking pattern analysis using CatWalk $X T^{\circledR}$ (C-H). Parameters analyzed include the regularity index (C) and the base of support (D). For paws located ipsilateral to the spinal cord lesion PRE-084 does not improve walking parameters such as the print position (E), the stride length (F) and the duty cycle (G). The paw angle of the hind paw located contralateral to the lesion is altered in the SCl-treated mice (H). Number of mice: Uninjured mice 6 (3 treated and three untreated, plain lines), hemisected mice 24 (12 treated and 12 untreated, dashed lines). Statistics: two-way ANOVA followed by Bonferroni tests in the comparison between injured control and injured treated groups (dashed lines), ${ }^{* *} p<0.01$.

Netherlands) were used to analyze statics and dynamics locomotor parameters as earlier described (Noristani et al., 2018). In brief, the open field test was used to assess spontaneous motor activity, animals were placed in an empty test arena $(50 \times 50 \mathrm{~cm})$ and their spontaneous motor activity was video recorded. Each analysis session lasted $10 \mathrm{~min}$ ( $2 \mathrm{~min}$ without recording followed by $8 \mathrm{~min}$ video recorded). Dynamic walking patterns analysis was done using CatWalk $\mathrm{XT}^{\circledR}$ through several parameters (including regularity index, base of support, print position, stride length, duty cycle and paw angle, Figures $\mathbf{1 C}-\mathbf{H}$ ). A minimum of five runs crossed at the same speed and at least 3-full step sequence patterns per run were analyzed. For injured mice, hind paw located on the ipsilateral side of SCI were not detectable by CatWalk before 14 days after injury, curves between 0 - and 14-days post injury were thus extrapolated (Figures 1C-G). 

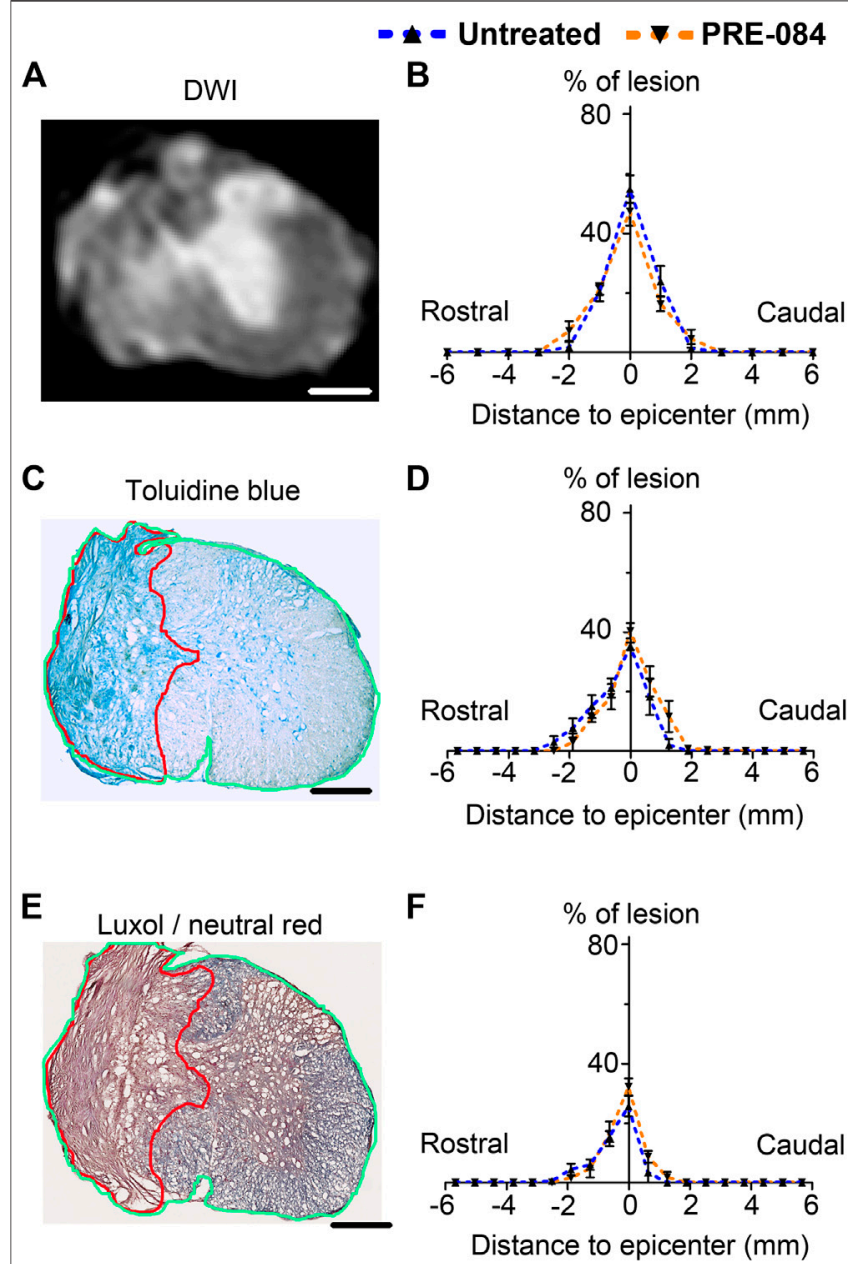

FIGURE 2 | Lesion size analysis. Representative ex vivo diffusion weighted MRI (DWI) (A), toluidine blue staining (C) and luxol fast blue/neutral red staining (E) at lesion epicenter 6 weeks after SCl. Quantifications of the lesion percentage at the epicenter, the lesion extension and the lesion volume represented by area under the curve using DWI acquisitions (B), toluidine blue sections staining (D) and luxol fast blue/neutral red (F) in both treated and untreated injured animals. Note the damaged spinal cord tissue (outlined in red in $\mathbf{C}$ and $\mathbf{E}$ ) used for lesion quantification and allows distinction from undamaged tissue (outlined in green in $\mathbf{C}$ and $\mathbf{E}$ ) Number of hemisected mice: DWI six treated and six untreated and histology five treated and five untreated. Scale bars: A, C and E $600 \mu \mathrm{m}$.

\section{Ex vivo Diffusion Magnetic Resonance Imaging}

Six weeks after SCI, mice were injected with a lethal dose of tribromoethanol (i.p., $500 \mathrm{mg} / \mathrm{kg}$, Sigma-Aldrich Darmstadt, Germany) and perfused intracardially with phosphate saline buffer (PBS, 0.1M, pH 7.2) followed by $4 \%$ paraformaldehyde (PFA, pH 7.2, Sigma Aldrich, Darmstadt, Germany) in 0.1M PBS. Spinal cords were post-fixed for $2 \mathrm{~h}$ in $4 \%$ PFA and stored in PFA $1 \%$ until MRI acquisition. MRI acquisition and analysis were done as previously described (Noristani et al., 2018). Briefly, spinal cords were positioned in custom-made solenoid coil designed for spinal cord acquisition (Coillot et al., 2016) and placed in a 9.4 $\mathrm{T}$ apparatus (Agilent Varian 9.4/160/ASR, Santa Clara, California, USA). Diffusion MRI parameters: delta $=$ $6.88 \mathrm{~ms}, \mathrm{G}=10 \mathrm{G} / \mathrm{cm}-1$, separation $=15.05 \mathrm{~ms}, \mathrm{TR}=1,580 \mathrm{~ms}$, $\mathrm{TE}=30.55 \mathrm{~ms}, \mathrm{AVG}=30, \mathrm{FOV}=10 \mathrm{~mm} \times 10 \mathrm{~mm}$, slices $=36$, thickness $=1 \mathrm{~mm}$ without gap and acquisition matrix $=128 \times 128$. Segmentations were done using Myrian software (Intrasense, Montpellier, France) to analyze the lesion area (\% of the total surface area), the lesion extension along the rostro caudal axis and the lesion volume (area under the curve) (Figure 2B).

\section{Histology}

Following ex vivo MRI, spinal cords were cryoprotected in 30\% sucrose solution, frozen in OCT (Sakura, Alphen aan den Rijn, Netherlands) and stored at $-20^{\circ} \mathrm{C}$. Spinal cords were used for histological and immunohistochemical analysis on $14 \mu \mathrm{m}$ thick axial cryosections as earlier described (Noristani et al., 2017). To confirm lesion extension, spinal cord sections were analyzed using toluidine blue and luxol fast blue/neutral red. Briefly, for toluidine blue staining sections were washed twice in $0.1 \mathrm{M}$ PBS then incubated $10 \mathrm{~min}$ in $0.1 \%$ toluidine blue (Sigma Aldrich, Gilligham, United Kingdom), then soaked $10 \mathrm{~s}$ in $100 \%$ ethanol and washed twice in xylene. For luxol fast blue/neutral red staining, spinal cord sections were placed $5 \mathrm{~min}$ in $95 \%$ ethanol and then incubated in $0.1 \%$ luxol fast blue $(12 \mathrm{~h}$, room temperature, mild shaking). Slides were soaked $1 \mathrm{~min}$ in milli Q water before to be placed for $1 \mathrm{~min}$ in $0.05 \%$ lithium carbonate and washed $1 \mathrm{~min}$ in tap water. Slides were further incubated $10 \mathrm{~min}$ in $0.5 \%$ neutral red solution, dehydrated $5 \mathrm{~min}$ in $100 \%$ ethanol and washed twice $10 \mathrm{~min}$ in xylene. For both staining, slides were coverslipped with Eukitt (Sigma Aldrich, Gilligham, United Kingdom) and imaged using NanoZoomer Digital Pathology (NDP) System Hamamatsu (Hamamatsu, Hamamatsu city, Japan). On both rostral and caudal sides of the lesion, 10 sections were analyzed at $630 \mu \mathrm{m}$ intervals using NDP view software. The lesion area was measured as \% of the total surface area, lesion volume was estimated by the area under the curve (Figures 2D,F).

Peroxidase immunohistochemistry was performed as described before using anti-glial fibrillary acidic protein (GFAP) (1:1,000, catalog number Z0334, Dako, Glostrup, Denmark), anti-IBA1 (1:1,000, catalog number 019-19741, Wako Pure Chemical Industries, Osaka, Japan), anti-TMEM 119 (1:5, generous gift from Ben Barres's lab Stanford University, California, USA) and anti-NeuN (1:400, catalog number MAB 377, Merck Millipore, USA) primary antibodies and corresponding peroxidase-conjugated or biotinylatedconjugated (for TMEM119) secondary antibodies (1:500, Jackson Immunoresearch, Stratech Scientific Ltd, Soham, United Kingdom). Amplification was done for TMEM 119 staining through addition of Avidin Biotin Complex solution (Vector Laboratories Ltd. Peterborugh, United Kingdom) diluted at 1:100 in $0.1 \mathrm{M} \mathrm{PBS}$ and incubated for $1 \mathrm{~h}$ at room temperature (RT). Sections were then rinsed in TRIS, pH 7.6 (Sigma-Aldrich Darmstadt, Germany) and protein expression was visualized using DAB peroxidase substrate kit (Vector Labs, Burlingame, USA). Nickel was added to DAB solution for TMEM119 staining. The reaction was stopped by $3 \times 10 \mathrm{~min}$ rinses in $0.1 \mathrm{M}$ TRIS. For 
immunohistochemical experiments, up to 18 sections per mouse were analyzed $(630 \mu \mathrm{m}$ intervals throughout a $1.2 \mathrm{~cm}$ spinal cord segment centered on the lesion site). Peroxidase immunostainings were done at the same time for all animals for a given protein. Staining intensities were evaluated by measuring relative optical density (OD) using ImageJ (National Institutes of Health, USA). For neuromuscular junction (NMJ) quantifications, $16 \mu \mathrm{m}$ transverse cryosections of gastrocnemius-soleus-plantaris muscular complex were proceeded for NMJ labeling using cholinesterase staining (Karnovsky and Roots, 1964). NMJs were quantified in every three sections.

\section{Statistics}

Two-way analysis of variance (ANOVA) with Bonferroni post hoc tests were used for behavioral analysis. Unpaired t-tests with Welch correction were used for MRI and histological analysis. Significance was accepted at $p \leq 0.05$. Data were analyzed using GraphPad Prism 5.0 (GraphPad Software, Inc., CA, USA) and expressed as the mean \pm standard error of the mean.

\section{RESULTS}

\section{o1R Agonist Treatment After Spinal Cord Injury Does Not Improve Motor Function and Preservation of Neuromuscular Junction Density}

To investigate whether activation of $\sigma 1$ receptor after SCI is beneficial to motor recovery, we administered an oral 6-weeks PRE-084-treatment $(1 \mathrm{mg} / \mathrm{kg} /$ day $)$ to mice starting immediately after thoracic nine lateral hemisection of the spinal cord. As a prerequisite we verified that all behavioral parameters were affected by the injury (Figures 1A-H). PRE084 did not modify the weight curves over the course of the experiment neither in uninjured nor injured mice (Figure 1A). We studied locomotion of the animal over the treatment period. PRE-084 did not improve the general spontaneous motricity analyzed by open field in both uninjured and injured groups (Figure 1B). The regularity index that reflects the number of normal step sequence patterns relative to the total number of paw placements (Figure 1C) and the base of support of the hind paws (average width between the paws, Figure 1D) were not improved in injured mice treated with PRE-084. Similarly, $\sigma 1 \mathrm{R}$ agonist treatment had no effect on the print position of paws on the ipsilateral side of the spinal cord lesion (corresponding to the distance between the position of the hind paw and the position of the previously placed front paw in the same step cycle, Figure 1E). Also, the stride length and duty cycle on the ipsilateral side (indicating the stance duration as a percentage of the duration of the step cycle) remained unchanged with PRE-084 treatment (Figures 1F,G). On the contralateral side, $\sigma 1$ receptor activation transiently worsened contralateral hind paw placement since the paw angle (angle between the paw axis and the body axis) differed more from preoperative value $(100 \%$ correspond to

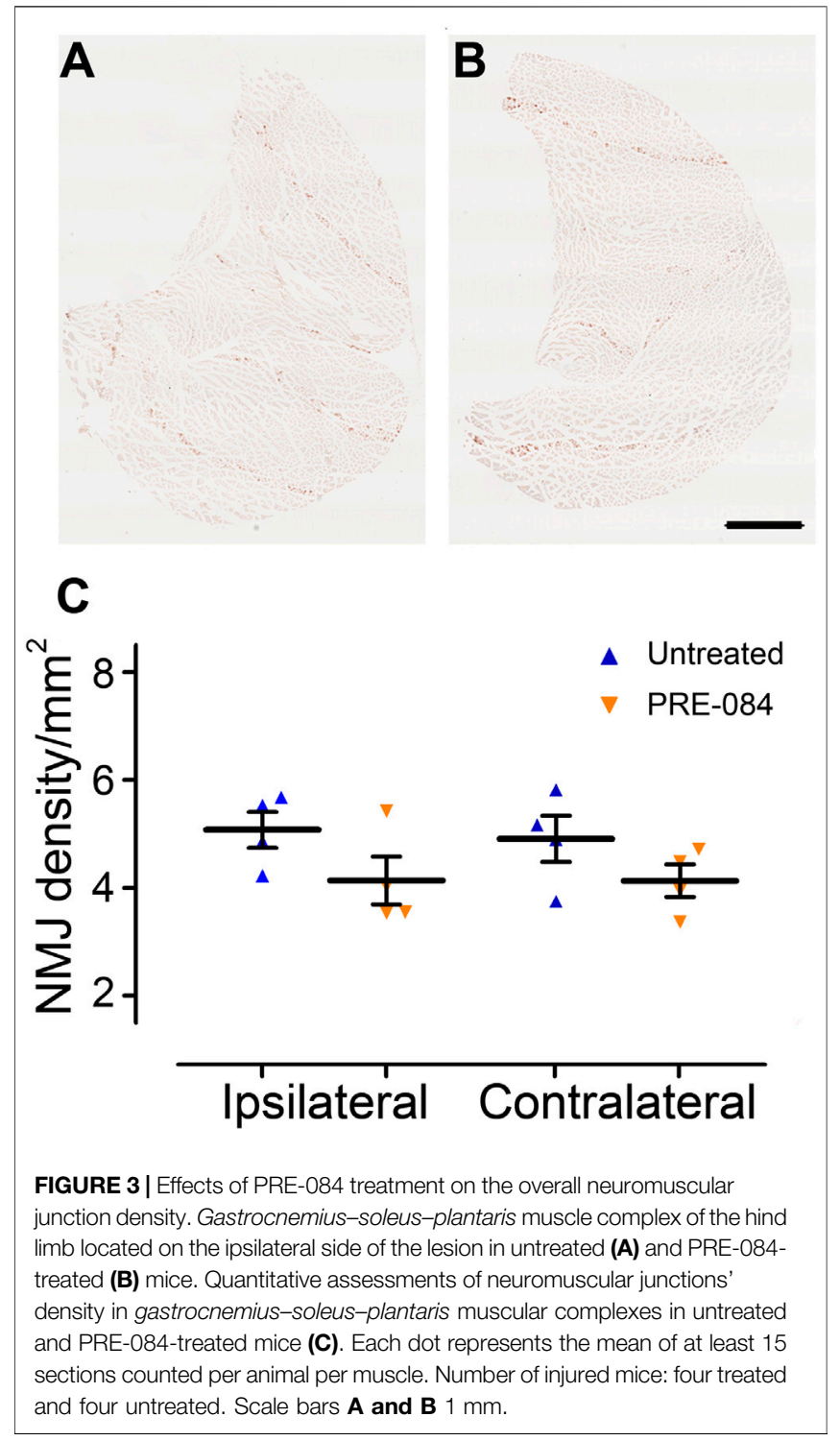

the angle prior to SCI) in the SCI-treated than in the SCIuntreated group (Figure 1H, $p=0.049$ ). Muscle alterations can affect paw angle placement, we thus investigated the gastrocnemius-soleus-plantaris complex (Figure $\mathbf{3}$ and Supplementary Figure 1A). No difference in number of neuromuscular junction densities in between groups was observed (Figures 3A-C, ipsilateral $p=0.15$; untreated: $5.07 \pm 0.33$; PRE-084: $4.14 \pm 0.45$ and contralateral $p=0.2$; untreated: $4.91 \pm 0.43$; PRE-084: $4.13 \pm 0.30$ ), however when taking into account all sections that were quantified (and not only the mean per animal), PRE-084-treatment decreased the neuromuscular junction densities in both hind limbs (Supplementary Figure 1A, $p<0.0001$ for both ipsilateral; untreated: $5.22 \pm 0.11$; PRE-084: $4.19 \pm 0.12$ and contralateral; untreated: $5.07 \pm$ 0.11; PRE-084: $4.00 \pm 0.09$ ).

Therefore, $\sigma$ 1receptor activation over a 6-weeks period starting immediately after SCI is slightly detrimental to motor function recovery in mice. 


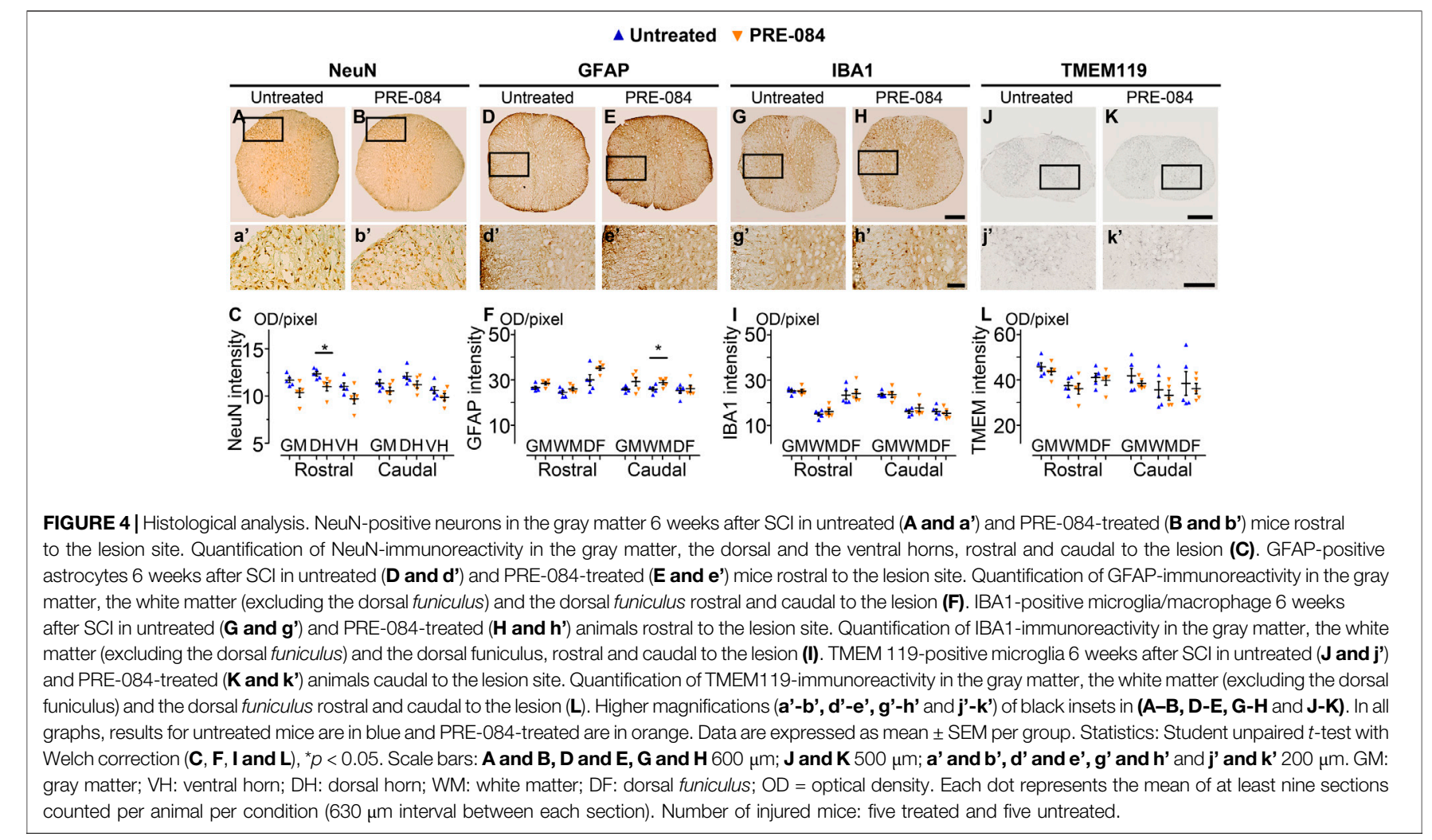

\section{o1R Agonist Treatment After SCI Decreases NeuN Expression and Increases Astrocytic Reactivity Without Limiting Lesion Size}

To analyze outcomes of $\sigma$ 1receptor activation on tissue structure following spinal cord lesion we acquired ex vivo DW-MRI (Figure 2A) and segmented $1 \mathrm{~mm}$-thick axial slices. Lesion extension on the rostro-caudal axis, lesion volume and percentage of damaged tissues at lesion epicenter were similar in both groups (Figure 2B). We further assessed the effect of PRE084 on spinal cord lesion size using classical histological methods such as toluidine blue (Figures 2C,D) and luxol fast blue/neutral red (Figures 2E,F) staining on the same spinal cord segment and confirmed the absence of difference between untreated and treated groups (Figures 2D,F). We then deepened our analysis and quantified the expression of neuronal (Figures $4 \mathbf{A}, \mathbf{B}$ and a' $\& \mathrm{~b}^{\prime}$ ) and glial markers (Figures 4D,E and d'\&e'; G\&H and g'\&h'; J\&K and $\left.j^{\prime} \& \mathrm{k}^{\prime}\right)$ on a $1.2 \mathrm{~cm}$-perilesional segment of the spinal cord using specific neuron (NeuN), astrocytes (GFAP), microglia and macrophages (IBA1) and microglia (TMEM 119) (Figures 4A-K) markers. We highlighted a decrease in NeuN expression in the treated group as compared to the untreated group in the dorsal horn of the rostral segment $(p=0.032$; untreated: $12.37 \pm$ 0.23; PRE-084: $11.00 \pm 0.43$ ) (Figure 4C), though when taking into account all sections that were quantified (and not only the mean per animal), PRE-084-treatment decreased NeuN expression in all regions (gray matter, ventral and dorsal horns in the rostral $(p<$ $0.0001)$ and caudal $(p<0.05)$ segments (Supplementary Figure 1B). GFAP expression was higher in the white matter of the treated group caudal to the lesion ( $p=0.048$; untreated: $25.94 \pm$ 0.82; PRE-084: $28.79 \pm 0.85$ ) (Figure 4F), similarly when taking into account all sections that were quantified, PRE-084-treatment increased GFAP expression in all regions rostral to the lesion $(p<$ 0.0001 ) and in the gray and the white matters (with the exception of the dorsal funiculus) caudal to the injury (Supplementary Figure 1C, $p<0.0001)$. No modulation of microglia/ macrophages was induced by $\sigma 1 \mathrm{R}$ agonist treatment. The overall IBA1 (Figure 4I and Supplementary Figure 1D) and TMEM119 (Figure 4L and Supplementary Figure 1E) expressions were similar in both groups.

Altogether, these results suggest that activation of $\sigma 1$ receptor using PRE-084 over 6 weeks after injury induces a decrease in $\mathrm{NeuN}$ expression and an increase in astrogliosis while microglia reactivity is not affected.

\section{DISCUSSION}

In this study, we assessed outcomes of sigma-1 receptor agonist treatment on functional recovery and tissue reorganization after SCI in mice. Our findings suggest a detrimental effect of PRE-084 post injury treatment on motor function recovery and is associated, at tissue level, with a decrease in NeuN expression and an increase in astrogliosis.

The involvement of $\sigma 1 \mathrm{R}$ in central neuropathic pain induced by SCI had been recently studied using both genetic and pharmacological approaches (Castany et al., 
2018; Castany et al., 2019). Following spinal cord contusion at thoracic level 8-9 mice lacking $\sigma 1 \mathrm{R}(\sigma 1 \mathrm{KO})$ display reduced injury-induced thermal hyperalgesia and mechanical allodynia. Moreover, $\sigma 1 \mathrm{KO}$ do not present an injuryinduced increase in spinal cord expression of the phosphorylated form of two molecules involved in central sensitization in neuropathic pain states i.e. extracellular signal-regulated kinases (ERK1/2) and NMDA receptor NR2B subunit. Conversely to wild type mice, SCI in $\sigma 1 \mathrm{KO}$ mice does not induce an upregulation of the pro inflammatory cytokines tumor necrosis factor (TNF- $\alpha$ ) and interleukin IL-1 $\beta$ (Castany et al., 2018). Mice treated with a selective $\sigma 1 \mathrm{R}$ antagonist starting either 1 month (Castany et al., 2018) or 1 week (Castany et al., 2019) following SCI, similarly display reduced induced-central neuropathic pain. No major modification of motor activity was observed in both treatment protocols. Moreover, $\sigma 1 \mathrm{R}$ antagonist treatment during the first week post SCI also prevented injuryinduced increases of pERK1/2, pNR2B-NMDA, TNF- $\alpha$ and IL-1 $\beta$ (Castany et al., 2019). In order to implement the two previous studies that investigated outcomes of a $\sigma 1 \mathrm{R}$ pharmacological modulation following SCI, we have chosen to perform our experiments in female mice. It had been shown a gender-dependent locomotor recovery following SCI; thus we cannot exclude a differential effect of sigma one agonist treatment in male mice (Farooque et al., 2006). Our findings, further suggest a detrimental effect of $\sigma 1 \mathrm{R}$ activation following SCI on motor function recovery that may thus result from an increased expression of proinflammatory molecules as reflected by an enhanced astrogliosis.

Sigma-1 receptor modulates calcium signaling between the endoplasmic reticulum and mitochondria and regulates mitochondrial functions, such as intramitochondrial $\mathrm{Ca}^{2+}$ homeostasis, reactive oxygen species (ROS) generation and cellular bioenergetics (Hayashi and Su, 2007). Mitochondrial dysfunction is an essential component of the secondary injury cascade induced by SCI. In particular, it leads to ATP loss and successive inactivation of ATP-dependent ion pumps required for regulation of ion concentrations and reuptake of the excitatory neurotransmitter glutamate. Moreover, a consequence of the persistent ion shift during secondary injury is an increased ROS. On the one hand, sigma-1 receptor agonists favor brain plasticity and induce protective effect in the CNS under pathological conditions (Maurice and Goguadze, 2017) but on the other hand isolated mouse brain mitochondria exposed to $\sigma 1 \mathrm{R}$ agonists display an increase in ROS level. Strikingly, PRE-084 induces a ROS increase of $24 \%$ associated with a $40 \%$ selective increase of complex one activity when applied to isolated mouse brain mitochondria (non-pathological condition) and decreases A $\beta 1-42$-induced ROS elevation (pathological condition i.e. amyloid toxicity) (Goguadze et al., 2019). Therefore, $\sigma 1 \mathrm{R}$ agonists exert condition-dependent effects on ROS production. The detrimental in vivo effect of per os PRE084 treatment over 6 weeks at this dose $(1 \mathrm{mg} / \mathrm{kg} /$ day $)$ following lateral hemisection of the spinal cord may thus be a consequence of an increased ROS level.
The present study therefore suggests the detrimental effect of $\sigma 1 \mathrm{R}$ agonist on motor recovery, neuronal preservation and astrogliosis following SCI. However, dose-dependent and time response effects of $\sigma 1 \mathrm{R}$ agonists and antagonists treatments in various animal models of traumatic CNS injury merits further investigation.

\section{DATA AVAILABILITY STATEMENT}

The raw data supporting the conclusions of this article will be made available by the authors, without undue reservation.

\section{ETHICS STATEMENT}

The animal study was reviewed and approved by the Veterinary Services Department of Hérault, the regional Ethic Committee $\mathrm{n}^{\circ} 36$, and the French Ministry of National Education, Higher Education, and Research (authorizations; mice: $n^{\circ} 34118$ ).

\section{AUTHOR CONTRIBUTIONS}

AL: investigation, GP: formal analysis, writing-original draft; SB: formal analysis. YG: conceptualization, investigation, formal analysis and supervision. FP: conceptualization, validation, visualization, project administration, funding acquisition and writing-review and editing.

\section{FUNDING}

This work was supported by the patient organizations "Demain Debout Aquitaine" (to YNG and FEP) and "Verticale" (to AL, YNG and FEP). The funding sources were not involved in study design, collection, analysis and interpretation of the data as well as in the writing of the report and in the decision to submit the article for publication.

\section{ACKNOWLEDGMENTS}

We thank Tangui Maurice for providing the PRE-084 and for sharing his expertize in sigma one receptor. We thank Ben Barres's lab (Stanford University, California, USA) for their generous TMEM119 antibody gift. We thank the BioNanoNMRI (University of Montpellier) for MRI acquisition and in particular Christophe Goze Bac and Maida Cardoso.

\section{SUPPLEMENTARY MATERIAL}

The Supplementary Material for this article can be found online at: https://www.frontiersin.org/articles/10.3389/fphar.2021.614949/ full\#supplementary-material. 


\section{REFERENCES}

Castany, S., Codony, X., Zamanillo, D., Merlos, M., Verdú, E., and Boadas-Vaello, P. (2019). Repeated sigma-1 receptor antagonist MR309 administration modulates central neuropathic pain development after spinal cord injury in mice. Front. Pharmacol. 10, 222. doi:10.3389/fphar.2019.00222

Castany, S., Gris, G., Vela, J. M., Verdú, E., and Boadas-Vaello, P. (2018). Critical role of sigma-1 receptors in central neuropathic pain-related behaviours after mild spinal cord injury in mice. Sci. Rep. 8, 3873. doi:10.1038/s41598-018-22217-9

Coillot, C., Sidiboulenouar, R., Nativel, E., Zanca, M., Alibert, E., Cardoso, M., et al. 2016). Signal modeling of an MRI ribbon solenoid coil dedicated to spinal cord injury investigations. J. Sens. Sens. Syst. 5, 137-145.doi:10.5194/jsss-5-137-2016

Farooque, M., Suo, Z., Arnold, P. M., Wulser, M. J., Chou, C. T., Vancura, R. W., et al. (2006). Gender-related differences in recovery of locomotor function after spinal cord injury in mice. Spinal Cord 44, 182-187. doi:10.1038/sj.sc.3101816

Goguadze, N., Zhuravliova, E., Morin, D., Mikeladze, D., and Maurice, T. (2019). Sigma-1 receptor agonists induce oxidative stress in mitochondria and enhance complex I activity in physiological condition but protect against pathological oxidative stress. Neurotox. Res. 35, 1-18. doi:10.1007/s12640-017-9838-2

Guzman-Lenis, M. S., Navarro, X., and Casas, C. (2009). Selective sigma receptor agonist 2-(4-morpholinethyl)1-phenylcyclohexanecarboxylate (PRE084) promotes neuroprotection and neurite elongation through protein kinase $\mathrm{C}$ (PKC) signaling on motoneurons. Neuroscience 162, 31-38. doi:10.1016/j.neuroscience.2009.03.067

Hall, A. A., Herrera, Y., Ajmo, C. T., Jr., Cuevas, J., and Pennypacker, K. R. (2009). Sigma receptors suppress multiple aspects of microglial activation. Glia 57, 744-754. doi:10.1002/glia.20802

Hayashi, T., and Su, T. P. (2007). Sigma-1 receptor chaperones at the ERmitochondrion interface regulate $\mathrm{Ca}(2+)$ signaling and cell survival. Cell 131, 596-610. doi:10.1016/j.cell.2007.08.036

Karnovsky, M. J., and Roots, L. (1964). A "Direct-Coloring" thiocholine method for cholinesterases. J. Histochem. Cytochem. 12, 219-221. doi:10.1177/12.3.219

Kumar, R., Lim, J., Mekary, R. A., Rattani, A., Dewan, M. C., Sharif, S. Y., et al. (2018). Traumatic spinal injury: global epidemiology and worldwide volume. World Neurosurg. 113, e345-e363. doi:10.1016/j.wneu.2018.02.033
Maurice, T., and Goguadze, N. (2017). Sigma-1 (sigma1) receptor in memory and neurodegenerative diseases. Handb. Exp. Pharmacol. 244, 81-108. doi:10.1007/ 164_2017_15

Motawe, Z. Y., Abdelmaboud, S. S., Cuevas, J., and Breslin, J. W. (2020). PRE-084 as a tool to uncover potential therapeutic applications for selective sigma-1 receptor activation. Int. J. Biochem. Cell Biol. 126, 105803. doi:10.1016/j. biocel.2020.105803

Noristani, H. N., Boukhaddaoui, H., Saint-Martin, G., Auzer, P., Sidiboulenouar, R., Lonjon, N., et al. (2017). A combination of ex vivo diffusion MRI and multiphoton to study microglia/monocytes alterations after spinal cord injury. Front. Aging Neurosci. 9. doi:10.3389/fnagi.2017.00230

Noristani, H. N., Saint-Martin, G. P., Cardoso, M., Sidiboulenouar, R., Catteau, M., Coillot, C., et al. (2018). Longitudinal MRI analysis and histological characterization after spinal cord injury in two mouse strains with different functional recovery: gliosis as a key factor. J. Neurotrauma 35 (24), 2924-2940. doi:10.1089/neu.2017.5613

Penke, B., Fulop, L., Szucs, M., and Frecska, E. (2018). The role of sigma-1 receptor, an intracellular chaperone in neurodegenerative diseases. Curr. Neuropharmacol. 16, 97-116. doi:10.2174/1570159X15666170529104323

Sha, S., Qu, W. J., Li, L., Lu, Z. H., Chen, L., Yu, W. F., et al. (2013). Sigma-1 receptor knockout impairs neurogenesis in dentate gyrus of adult hippocampus via down-regulation of NMDA receptors. CNS Neurosci. Ther. 19, 705-713. doi:10.1111/cns.12129

Conflict of Interest: The authors declare that the research was conducted in the absence of any commercial or financial relationships that could be construed as a potential conflict of interest.

Copyright $\odot 2021$ Lattard, Poulen, Bartolami, Gerber and Perrin. This is an openaccess article distributed under the terms of the Creative Commons Attribution License (CC BY). The use, distribution or reproduction in other forums is permitted, provided the original author $(s)$ and the copyright owner(s) are credited and that the original publication in this journal is cited, in accordance with accepted academic practice. No use, distribution or reproduction is permitted which does not comply with these terms. 